\title{
COVID 19 Infection amongst Healthcare Providers: Saviors or Survivors!
}

\author{
Parul Punia, Shuchi Mehra, Naina Nehra, Dhruva Chaudhary, Aparna Parmar, \\ Vinay Kumar, Suman Rangi, Sudesh Bala, Anjana Kumari and Davender Kumar*
}

Department of Orthodontics and Dentofcaial Orthopedics, Faculty of Pt. B. D Sharma, PGIDS, University of Health Sciences, Rohtak-124001, Haryana, India

*Corresponding author

Keywords

SARS CoV-2, COVID units, Non COVID units,

Health care providers (HCPs), Asymptomatic/

Presymptomatic patients, Screening

Article Info

Accepted:

12 May 2021

Available Online:

10 June 2021

\section{A B S T R A C T}

Since the emergence of COVID 19 pandemic, a huge population all across the world has suffered both in terms of morbidity and mortality. The health care providers (HCPs), who are the only armors against this virus, are at a greater risk of getting infected than the general population. To identify various risk factors contributing to the spread of SARS CoV-2 virus amongst doctors and nurses working in COVID and non COVID units. A prospective observational study over a period of five months was conducted on all COVID 19 infected HCPs (doctors and nurses). They were interviewed telephonically by the Infection Control Team using a standard set of questions based on infection control practices, travel history and contact exposure with confirmed or suspected personnel. The study included 192 HCPs infected with COVID 19. Out of these, 144 were doctors and 48 were nurses. A total of 41 HCPs were from the COVID units and 151 were from the non COVID units. In the non COVID unit, 103 had history of exposure to asymptomatic patients, while 48 got exposed through family, friends or colleagues. Most doctors in non COVID area were from the clinical departments. Doctors and nurses working in the COVID areas are more vigilant and careful as compared to those in non COVID units. The spurt in cases coincides with the peak observed in the community, hence all patients should be screened on admission and there must be regular screening amongst the HCPs.

\section{Introduction}

Severe acute respiratory Corona virus (SARS CoV-2) outbreak was declared as a global pandemic by WHO on $11^{\text {th }}$ March, 2020. ${ }^{1}$ As of $18^{\text {th }}$ October, a total of $39,774,852$ cases with $1,110,902$ deaths have occurred all over the world. ${ }^{2}$ With the community transmission being suspected in some parts of India, the figures are expected to rise exponentially with time. In many countries complete lock down was imposed, but the health care providers (HCPs) all across the globe were bound by their un-deterring sense of duty to serve the ailing mankind and were aggressively engaged in finding and treating the COVID 19 suspected cases. Nevertheless, these 'frontline workers' themselves are at a greater risk of 
getting infected and the burden of COVID-19 seems to be larger on the HCPs than the general population because of being frequently in contact with affected patients. In most countries, the rate of infection with COVID 19 amongst the healthcare workers (HCWs) has been very high and with significant mortality. ${ }^{3,4,5}$ Furthermore, there is always a risk of the infected health personnel thus transmitting the disease to the vulnerable patients, as social distancing measures are sometimes not possible while dealing with the patients.

Health care providers being our only saviors against this global war, their safety must be of highest priority and thus, it is imperative to find out various reasons behind the transmission of this virus amongst them. Few studies have attributed diverse factors such as inadequate personal protective equipment, work overload, insufficient diagnostic testing and exposure to infected patients as the risks for infection in healthcare personnel. ${ }^{6,7,8}$

Such data from India are scarce and to the best of our knowledge, no such studies evaluating the risk factors contributing to the COVID 19 infections amongst HCPs have been assessed. Hence this study was planned to evaluate the possible source of exposure and transmission of COVID-19 virus amongst HCPs working in COVID-19 units as well as non-COVID-19 areas.

\section{Materials and Methods}

\section{Study setting}

At our institute, we have three dedicated COVID units with a total capacity of 179 beds and two dedicated COVID ICUs with 31 beds. The resident doctors or nurses were posted in the COVID wards only after meticulous infection prevention and control training considering donning, doffing, bio medical waste management and disinfection protocols. For duties in the COVID wards, they were provided with complete PPE including coverall, headcaps, N-95 respirators, face shield and shoe covers.

All HCPs doing duties in the COVID wards were tested as part of routine screening done within 4-5 days of completion of COVID duties to rule out any asymptomatic infection.

HCPs doing duties in non COVID wards got tested if and when they developed signs and symptoms suggestive of COVID 19 or after any exposure to some suspected or confirmed COVID 19 patient/ colleague/ family member.

After complete lockdown in India on $24^{\text {th }}$ March 2020, some OPD services of our institute were also suspended, while in others, number of patients was restricted. Rest emergency OT and other emergency services were running during this period. The OPD resumed functioning on $15^{\text {th }}$ July, 2020.

\section{Study design}

A prospective observational study over a period of five months $\left(1^{\text {st }}\right.$ May to $30^{\text {th }}$ September) was conducted on all HCPs (doctors and nurses) diagnosed with COVID 19 viral infection to analyze the potential risk factors associated with the transmission of this virus in the health care facility.

The diagnosis of COVID 19 for all HCPs was based on by RT-PCR for SARS CoV-2 using nasopharyngeal and oropharyngeal swabs. All the COVID 19 infected doctors and nurses were interviewed telephonically by the Infection Control Team using a standard set of questions based on infection control practices, travel history and contact exposure with confirmed or suspected personnel over the past two weeks preceding sign and symptom onset. Each participant was informed about 
the study purpose and a verbal consent was taken before proceeding with the telephonic interview and confidentiality of the HCPs was maintained. A total of 152 doctors and 48 nurses had been infected till $30^{\text {th }}$ September. Out of them, 8 health personnel could not be contacted for various reasons such as not picking up calls or wrong numbers provided to us.

\section{Results and Discussion}

The first patient of COVID 19 came to our institute on $23^{\text {rd }}$ March. The first HCW who came COVID 19 positive was posted in one of the COVID wards, on $10^{\text {th }}$ May. Since that day and till $30^{\text {th }}$ September, we were able to contact 192 HCPs (doctors and nurses) who had been infected with COVID 19. Out of these, 144 were doctors and 48 were nurses. Out of 192 doctors and nurses, $21.3 \%$ developed infection from the COVID units. Amongst the doctors, 23 developed COVID 19 infection during or 4-5 days post their duties in COVID wards/ICUs and 94 developed the infection from non COVID wards (Fig.1). Amongst these 16, fourteen were posted in COVID ICU, rest nine in COVID wards. Also, out of the 9 posted in COVID wards, 2 stayed in hostels during their duty and 2 went on home quarantine instead of institutional quarantine.

Amongst the 121 doctors from non COVID units, the probable source of infection in 77 doctors was contact with unsuspected COVID patient who later was diagnosed as COVID positive.

In the remaining 44, the possible source of infection that could be elicited was while dining with asymptomatic friends/ family/ colleagues or staying in the same hostel or premises. (Fig.2). Amongst those who turned out to be COVID positive, the initial reason for getting COVID test done was as a result of development of symptoms in 114 and as a part of routine screening or as part of contact tracing in rest of 20 doctors. (Fig. 3)

The number of doctors infected while working in the non COVID areas from different departments is given in Table I.

Amongst the nurses who came COVID positive, 18 were from the COVID areas and 30 were from the non COVID wards. (Fig.1) Out of these 18 nurses from the COVID ward, 2 had a history of attending a social gathering where few people were later found to be infected with COVID $19.90 \%$ of the nurses coming COVID positive from non COVID wards had history of coming in contact with unsuspected asymptomatic/ presymptomatic patients. (Fig.5) Amongst the nurses, 33 got tested due to the development of symptoms and rest 15 got tested as part of routine screening. (Fig.3) Month wise distribution of doctors and nurses coming COVID positive is shown in Fig. 4

\section{Non COVID areas}

In our study, nearly $80 \%$ of the doctors and nurses who acquired SARS CoV-2 infection were from the non-COVID areas, i.e, working in OPDs, emergencies, labor room, etc. Although universal masking is being implemented, but in non COVID units, the healthcare staff as well as patients have a laid back attitude towards COVID-19 transmission and are thus barely compliant with infection control protocols. In a study done in Cleveland, it was observed that all high risk exposures were from the non COVID area and not even a single HCW working in COVID 19 areas got infected. ${ }^{10}$

The predominant mode of infection amongst these healthcare workers in our institute was direct exposure to unknown COVID-19 asymptomatic or pre-symptomatic patients in 
non COVID wards/OPDs. The delayed diagnosis in these unsuspected patients contributes to the major mode of transmission amongst healthcare workers. Various studies have documented the transmission of SARS CoV-2 virus via asymptomatic and presymptomatic individuals who pose a much greater risk for the spread of this disease. ${ }^{11,12,13}$ During the lockdown, the number of HCPs infected at our institute was meager. Once the OPD's and other routine services resumed after lockdown, there was a gradual increase in the number of HCPs infected with COVID 19, implying community transmission via asymptomatic/pre-symptomatic unsuspected patients. Henceforth, it becomes critical to ensure surveillance through routine testing of all admitted patients to rule out any asymptomatic transmission. In the study done in Cleaveland, it was observed that the risk exposure to such unsuspected patients was strikingly brought down as the policy of routine admission screening amongst patients for SARS CoV-2 virus was implemented. ${ }^{10}$ The spurt in COVID 19 cases amongst HCPs at our institute was observed two weeks earlier in mid August as compared to September in general population in Rohtak district. Better awareness, routine screening post COVID duty and meticulous contact tracing in healthcare settings led to prior detection of the peak observed in cases.

The maximum number of cases in the non COVID area was observed from the obstetrics \& gynecology and dental department. In the labor room, this could be attributed to patient overload, long duty hours, inability of patients in labor to wear mask, rising number of asymptomatic and pre-symptomatic cases with unknown COVID status in the community. Hence, it is particularly important to screen pregnant mothers on admission. Dentists have to work in very close contact with patients, so it is imperative to screen the patients before attending to them. More than $90 \%$ of the doctors infected were from the clinical departments. Due to execution of virtual classes and less patient exposure in paraclinical departments, the risk of getting exposed is drastically reduced.

Another salient mode of transmission of this virus amongst HCP from the non COVID areas had been the doctors residing in the hostels who rarely adhere to measures like social distancing and universal masking.

Few workers from the hostel mess who were asymptomatic initially also turned out to be COVID positive later on. Colleagues and hostellers dine together for which they tend to remove their masks, favoring the shedding of virus in each other's vicinity. In present-day times, when the COVID cases are on a rise in the community, it is likely that the hospitals become hubs for sustained viral transmission, hence we advocate widespread screening of all HCPs. Few studies suggested that if testing capacity allows reporting in real time, routine testing of asymptomatic HCWs could reduce onward transmission by $16-23 \% .{ }^{14,15,}{ }^{16}$ For routine screening, the rapid antigen detection kits may also be used but their sensitivity and specificity must be evaluated.

The clinical presentation of COVID 19 is variable and asymptomatic cases account for nearly $10-30 \%$ of the cases. ${ }^{17,18}$ Since everyone at our institute was not screened, the total number of asymptomatic cases may not be predicted. $74.5 \%$ of COVID 19 positive HCPs in our institute got themselves tested due to the onset of signs and symptoms suggestive of COVID 19 including fever, myalgia and sore throat as the commonest ones. Rest got tested as a part of routine screening after their COVID duties. 
Table.1 Department wise distribution of COVID 19 infected doctors from non COVID areas

\begin{tabular}{|c|c|c|c|c|c|}
\hline Department & Number & Department & Number & Department & Number \\
\hline Gynaecology & 19 & Pathology & 5 & Pharmacology & 2 \\
\hline Dentistry & 17 & Chest and T.B & 4 & Physiology & 2 \\
\hline Peadiatrics & 11 & PSM & 4 & Administrative Block & 3 \\
\hline Anaesthesia & 10 & Gastroentorology & 2 & Nephrology & 1 \\
\hline Orthopedics & 10 & Sports Medicine & 2 & Dermatology & 1 \\
\hline Surgery & 8 & Radiotherapy & 2 & ENT & 1 \\
\hline Medicine & 7 & $\begin{array}{c}\text { Pulmonary critical } \\
\text { care medicine }\end{array}$ & 2 & & \\
\hline Radiology & 5 & Ophthalmology & 2 & & \\
\hline
\end{tabular}

Fig.1 Number of HCPs infected from COVID and Non COVID areas. (Doctors and Nurses)

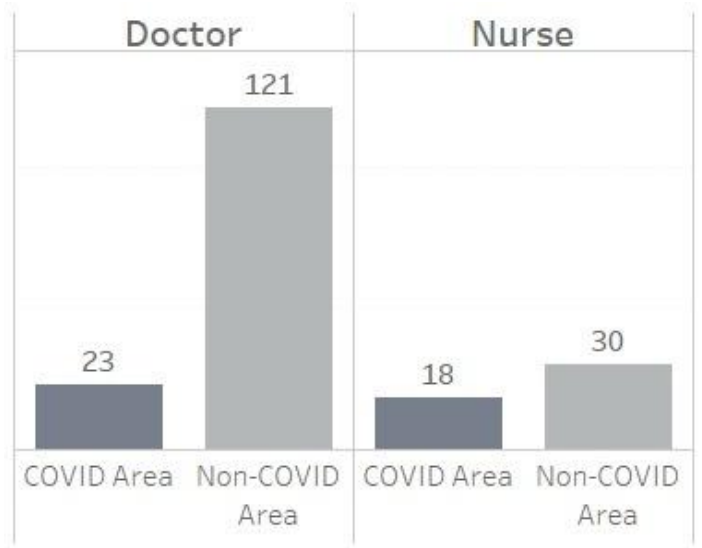

Fig.2 Probable source of infection in COVID 19 infected HCPs (Doctors and Nurses)

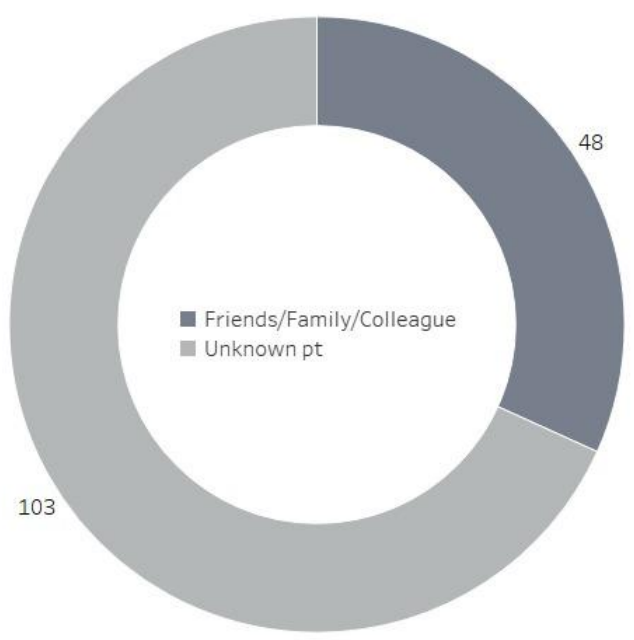


Fig.3 Number of asymptomatic and symptomatic COVID infected HCPs (Doctors and Nurses)

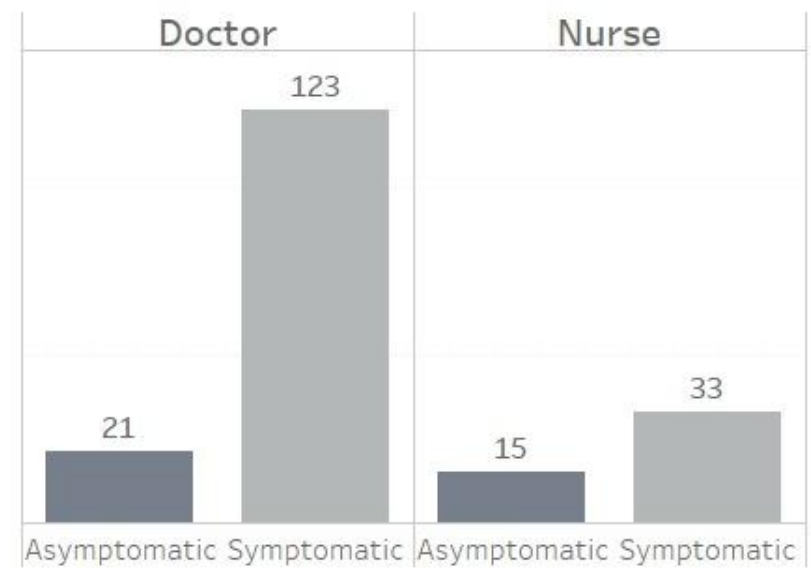

Fig.4 Month wise distribution of COVID 19 infected HCPs and general population in Rohtak ${ }^{9}$

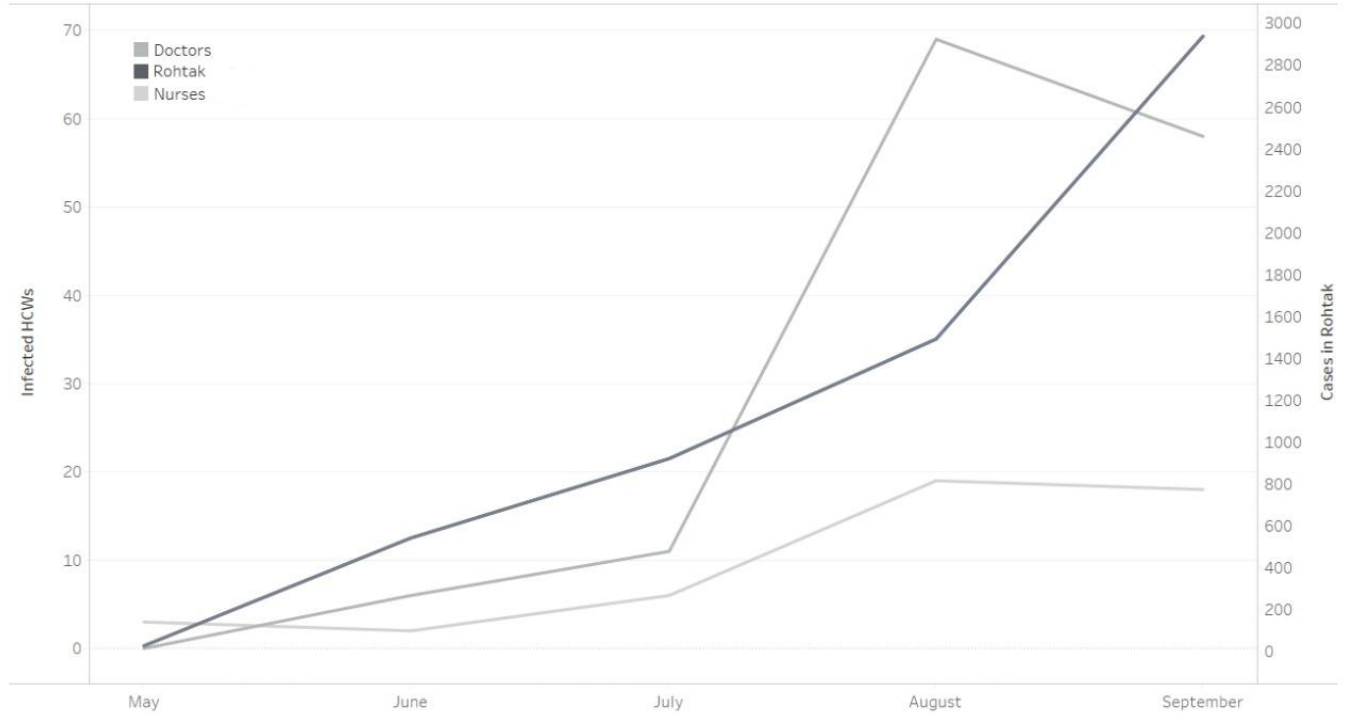

\section{COVID areas}

Out of 23 infected doctors working in COVID areas, nine had been from COVID wards and fourteen from the COVID ICUs. Amongst the 9 doctors with duties in the COVID wards, 2 had been on home quarantine and another 2 stayed in hostels during their duty days, so the exact source of infection in these 4 individuals cannot be ascertained.

In the COVID ICUs, there is more probability of prolonged close contact with the patients which puts the HCPs at increased risk of exposure due to various aerosol generating procedures such as endotracheal intubation and respiratory suctioning. To provide the doctors with the best level of protection, WHO recommends the presence of negative pressure ICUs with natural ventilation to prevent the spread of airborne pathogens. ${ }^{19}$

Some residents faced practical issues such as mask getting spoiled due to sweating thus impeding its viability or urge to adjust PPE while on long duty hours. These issues need to 
be addressed during the training of infection control practices to all HCPs before they gear up for their COVID duties. The marginal number of COVID positive doctors and nurses working in COVID areas augments the significance of infection control training imparted to them. Nevertheless, even with all infection prevention and control precautions, infection in the HCPs working in COVID 19 areas is inevitable as has been highlighted in other studies as well. ${ }^{20}$

The present study had few limitations. It was based on telephonic communication so interviewer and interviewee bias may not be ruled out sometimes.

Our study highlights the importance of implementing stringent infection control practices including universal masking and social distancing, especially in the non COVID settings by creating awareness regarding asymptomatic or pre-symptomatic transmission of cases. We advocate mass screening amongst doctors especially those residing in the hostels and other such locations to identify the asymptomatic HCPs who can be timely isolated to prevent further transmission to other HCPs as well as patients. Screening of patients on admission should also be given prime importance owing to the possible community transmission.

\section{Salient Points}

Healthcare professionals working in the non COVID-19 units are getting infected more as compared to COVID units.

No HCP should be posted in any COVID unit before stringent infection control practices training.

Asymptomatic/pre-symptomatic patients contribute as the major risk of exposure to the HCPs.
Asymptomatic / pre-symptomatic doctors and nurses also pose a threat to the spread of COVID 19.

All admitted patients should be screened before admission.

Widespread routine screening must be done in all HCPs.

There is a possibility of community transmission occurring in Rohtak distt.

\section{Acknowledgements}

We would like to thank and acknowledge The Medical Superintendent Office, PGIMS, Rohtak for permitting us to conduct the study and The Control Room for COVID-19, PGIMS Rohtak for providing us the data.

\section{References}

1. World Health Organization (2020). Coronavirus disease (COVID-19) Pandemic, WHO. Accessed from https://www.who.int/emergencies/disease s/novel-coronavirus-2019.

2. World Health Organization. Coronavirus disease (COVID-2019) situation reports. $2020 . \quad$ https ://www.who.int/emergencies/diseases/nov el-coronavirus-2019/situation-reports.

3. T M McMichael, D W Currie, S Clark, S Pogosjans, M Kay, N G Schwartz, et al., Epidemiology of Covid-19 in a LongTerm care facility in king county, Washington. N. Engl. J. Med 2020; 382(21):2005-11.

DOI: https://doi.org/10.1056/NEJMoa2005412, PMID: 32220208

4. Pathak B G, Manapurath R M. Combating the pandemic of COVID-19 in India: health care worker perspective. Int $\mathrm{J}$ Community Med Public Health 2020;7(7):2797-802 
5. Maltezou H C, Dedoukou X, Tseroni M, Tsonou E, Raftopoulos V, Papadima K, et $a l$., SARS-CoV-2 Infection in healthcare personnel with high-risk occupational exposure: evaluation of seven-day exclusion from work policy. Clin Infect Dis. 2020 Jun 29:ciaa888.https://doi.org/ 10.1093/cid/ciaa888. Epub ahead of print. PMID: $\quad 32594160 ; \quad$ PMCID: PMC7337654.

6. Characteristics of health care personnel with COVID-19 - United States, February 12 - April 9, 2020. MMWR Morb Mortal Wkly Rep. 2020;69:477-81.

7. Roxby A C, Greninger A L, Hatfield K M, Lynch J B, Dellit T H, James A, et al., Outbreak investigation of COVID-19 among residents and staff of an independent and assisted living community for older adults in Seattle, Washington [e-pub ahead of print]. JAMA Intern Med. 2020 Aug $1 ; 180(8): 1101-5$.

https://doi.org/10.1001/jamainternmed.20 20.2233. PMID: 32437547; PMCID: PMC7292007.

8. Mani N S, Budak J Z, Lan K F, BrysonCahn C, Allison Zelikoff, Gwendolyn E C Barker, et al., Prevalence of COVID-19 infection and outcomes among asymptomatic healthcare workers in Seattle, Washington [e-pub ahead of print]. Clin Infect Dis. https://doi.org/10.1093/cid/ciaa761.

9. Covid 19 Sero Survey Report of Haryana. Accessed from: http:/www.nhmharyana.gov.in/

10. Zabarsky T F, Bhullar D, Silva S Y, Mana B S, Ertle R N, Navas M D, et al., American Journal of Infection Control 00 (2020) 1-4.

https://doi.org/10.1016/j.ajic.2020.08.004

11. Huff H. V. and Singh A. Asymptomatic transmission during the COVID-19 pandemic and implications for public health strategies. Clin Infect Dis. 2020
May 28:ciaa654. doi: 10.1093/cid/ciaa654. Epub ahead of print. PMID: $\quad 32463076$; PMCID: PMC7314132.

12. Rivett L, Sridhar S, Sparkes D, Routledge M, Jones N K, Forrest S, et al., Screening of healthcare workers for SARS-CoV-2 highlights the role of asymptomatic carriage in COVID-19 transmission. Elife. 2020 May 11;9: e58728. doi: 10.7554/eLife.58728. PMID: 32392129; PMCID: PMC7314537.

13. Wong J, Jamaludin S A, Alikhan M F, Chaw L. Asymptomatic transmission of SARS-CoV-2 and implications for mass gatherings. J. Influenza Other Respir Viruses. 2020 ;14(5):596-8. https://doi.org/10.1111/irv.12767

14. Black J R M, Bailey C, Przewrocka J, Dijkstra K K, Swanton C. COVID-19: the case for health-care worker screening to prevent hospital transmission. The Lancet. 2020;395:1418-20. doi: 10.1016/S0140-6736(20)30917-X

15. M Gandhi, D S Yokoe and D V Havlir. Asymptomatic Transmission, the Achilles' Heel of Current Strategies to Control Covid-19. N Engl J Med 2020;382:215860

16. Imperial College COVID-19 Response Team. 2020. Report 16: role of testing in COVID-19 control. https://www.imperial.ac.uk/media/imperia 1-college/medicine/mrc-gida/2020-04-23COVID19-Report-16.pdf

17. He X, Lau E H Y, Wu P, Deng X, Wang $\mathrm{J}$, Hao $\mathrm{X}$, et al., Temporal dynamics in viral shedding and transmissibility of COVID-19. Nature Medicine 2020;26:672-5.

DOI: https://doi.org/10.1038/s41591-0200869-5, PMID: 32296168

18. Gao Z, Xu Y, Sun C, Wang X, Guo Y, Qiu S, Ma K. A Systematic Review of Asymptomatic Infections with COVID19. J Microbiol Immunol Infect. 2020 
May 15. doi: 10.1016/j.jmii.2020.05.001. Epub ahead of print. PMID: 32425996; PMCID: PMC7227597.

19. Shang Y, Pan C, Yang X, Zhong M, Shang $\mathrm{X}, \mathrm{Wu} \mathrm{Z}$, et al., Management of critically ill patients with COVID 19 in ICU; statement from front line intensive care experts in Wuhan, China. Ann. Intensive Care. 10, 73 (2020). https://doi.org/10.1186/s13613-020-

00689-1

20. Nguyen L H, Drew D A, Joshi A D, Guo C G, Ma W, Mehta RS et al.,. Risk of COVID-19 among frontline healthcare workers and the general community: a prospective cohort study. medRxiv [Preprint]. $2020 \quad$ May 25:2020.04.29.20084111. doi: 10.1101/2020.04.29.20084111.

\section{How to cite this article:}

Parul Punia, Shuchi Mehra, Naina Nehra, Dhruva Chaudhary, Aparna Parmar, Vinay Kumar, Suman Rangi, Sudesh Bala, Anjana Kumari and Davender Kumar. 2021. COVID 19 Infection amongst Healthcare Providers: Saviors or Survivors!. Int.J.Curr.Microbiol.App.Sci. 10(06): 8290. doi: https://doi.org/10.20546/ijcmas.2021.1006.008 